\title{
Aromaticity of acenes: the model of migrating circuits.
}

\author{
Dariusz W. Szczepanik, ${ }^{* 1}$ Miquel Solà, ${ }^{2}$ Tadeusz M. Krygowski, ${ }^{3}$ Halina Szatylowicz, ${ }^{4}$ Marcin \\ Andrzejak, ${ }^{1}$ Barbara Pawełek, ${ }^{5}$ Justyna Dominikowska, ${ }^{6}$ Mercedes Kukułka, ${ }^{1}$ and Karol Dyduch. ${ }^{1}$ \\ ${ }^{1}$ K. Gumiński Department of Theoretical Chemistry, Faculty of Chemistry, Jagiellonian University, Gronostajowa 2, 30-387 \\ Kraków, Poland. \\ ${ }^{2}$ Institut de Quìmica Computacional i Catàlisi, Universitat de Girona, C/ Maria Aurèlia Capmany, 69, 17003 Girona, Catalo- \\ nia, Spain. \\ ${ }^{3}$ Department of Chemistry, University of Warsaw, Pasteura 1, 02-093 Warszawa, Poland. \\ ${ }^{4}$ Faculty of Chemistry, Warsaw University of Technology, Noakowskiego 3, 00-664 Warszawa, Poland. \\ ${ }^{5}$ Department of Plant Cytology and Embryology, Institute of Botany, Jagiellonian University, Gronostajowa 9, 30-387 Kra- \\ ków, Poland. \\ ${ }^{6}$ Theoretical and Structural Chemistry Group, Faculty of Chemistry, University of Lodz, Pomorska 163/165, 90-236 Łódź, \\ Poland.
}

\begin{abstract}
The concept of migrating Clar's sextet is extended to explain the local aromaticity trends in linear acenes predicted by different aromaticity criteria from theoretical calculations as well as from experimental data. The electron density of delocalized bonds is used to assess the link between resonance and reactivity and to rationalize the constant-height AFM image of pentacene.
\end{abstract}

The aromatic $\pi$-sextet rule, originally proposed by Erich Clar in $1972,{ }^{1}$ allows one to rationalize reactivity of a great number of polybenzenoid hydrocarbons by qualitative assessment of their global and local aromatic character. ${ }^{2}$ It states that the electronic structure of a polycyclic benzenoid system is predominated by the so called Clar structure, i.e. the Kekulé resonance form containing the largest possible number of disjoint aromatic $\pi$-sextets (i.e. fully conjugated benzene-like moieties). Accordingly, benzenoid species can be divided into three groups: (I) those containing only aromatic $\pi$-sextets and Clar's 'empty' rings, e.g. triphenylene; (II) those that have $\pi$-sextets and rings with only one double bond, e.g. phenanthrene; (III) and those containing rings with two double bonds, e.g. anthracene. ${ }^{2}$ Benzenoid systems from the first two groups are characterized by a unique Clar structure with well-localized $\pi$-sextets and their physical and chemical properties have been proved experimentally and theoretically to be well-explained by Clar's aromatic $\pi$-sextet rule. ${ }^{2}$ Contrariwise, species from the third group are usually described by a superposition of equivalent Clar structures, giving rise to the so called 'migrating' $\pi$-sextet. In such cases the original formulation of the aromatic $\pi$-sextet rule may not give a clear answer to which ring is more stabilized than the other (each position of the migrating $\pi$-sextet is equally likely). ${ }^{2}$ Probably the best example is the class of $[n]$ acenes $(n=3,4,5)$ as they have been a subject of heated debate in literature for a long time ('the anthracene problem') ${ }^{3}$ owing to discrepancies between the groundstate aromaticity descriptions provided by Clar's rule (equally aromatic rings), ${ }^{2}$ quantitative studies involving different local aromaticity criteria (inner rings more aromatic than the outer ones or the contrary), ${ }^{3,4}$ and the experimentally assessed reactivity (inner rings more reactive towards addition and Diels-Alder reactions). ${ }^{5}$ In this work we reconcile these seemingly conflicting descriptions by introducing a simple extension of the qualitative concept of Clar's migrating $\pi$-sextet and we use it to rationalize the relationship between local aromaticity and the actual reactivity of anthracene, tetracene, and pentacene.

One of the fundamental limitations of Clar's rule is that it excludes from considerations any other fully-conjugated circuits except $\pi$-sextets $(6 \pi)$, thus being unable to describe the resonance effects between adjacent benzenoid units, i.e. the inter-ring resonance. Consequently, the predicted global aromaticity of the polybenzenoids may be systematically underestimated (especially for systems from the third group of Clar's classification). ${ }^{6}$ Moreover, it has been shown many times by statistical analyses that by taking into account higher $\pi$-electron circuits, i.e. $\pi$-dectets $(10 \pi)$, $\pi$-tetradectets $(14 \pi)$, etc., one can explain most of the discrepancies between local aromaticity descriptors based on magnetic, structural, and electronic criteria. ${ }^{7}$ Generally, there is no universal method to determine contributions from higher $\pi$-electron circuits and thus to investigate the inter-ring resonance effects in polycyclic benzenoid systems. ${ }^{6}$ Linear acenes, however, are very specific in this context, because here $\pi$-sextets may 'migrate' throughout the entire system, which results in the (incorrect) uniform-ratio patterns of relative local aromaticity - see the first column in Figure 1. To include explicitly the inter-ring resonance effects in acenes, a natural extension of the original Clar's concept has to be introduced that considers similar positional isomerism for higher-order circuits - see the second and third columns in Figure 1. In contrast to the benzene-like moieties, superposition of the isomeric structures of fully conjugated naphtha- and anthracyclic units introduces diversity into the relative ring aromaticity. Furthermore, as shown in the case of pentacene, the larger the migrating $\pi$-electron circuit, the greater the diversity in the resulting pattern. The number of resulting motifs with different ratios equals exactly the number of symmetry unique ring positions in each acene. For instance, in the case 


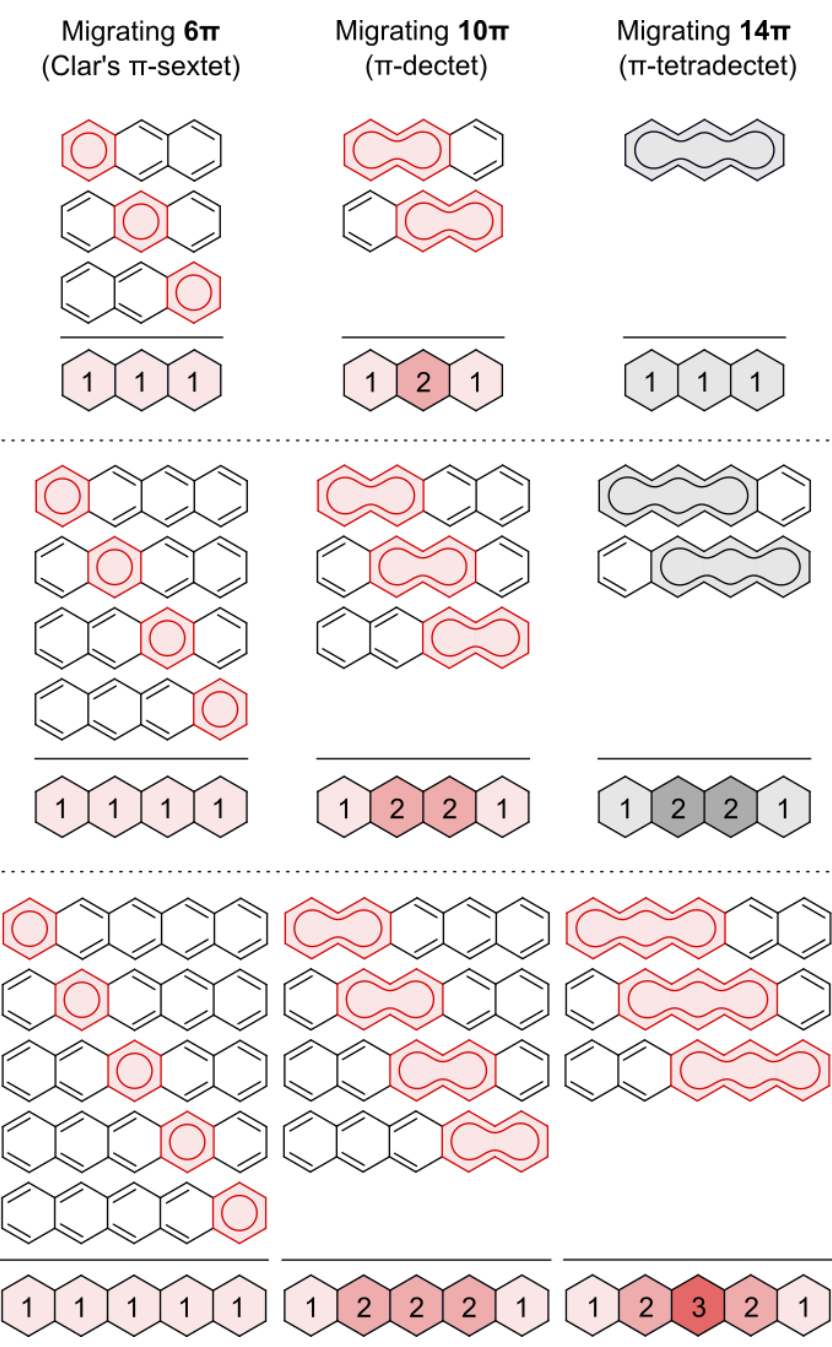

Figure 1. Schematic representation of migrating $\pi$-electron sextets, dectets, and tetradectets in [ $n]$ acenes, $n=3,4,5$, with the resulting local aromaticity ratios.

of anthracene with two unique rings there are only two linearly independent patterns, i.e. $(1: 1: 1)$ and $(1: 2: 1)$, resulting from migration of $\pi$-electron sextet and dectet, respectively; here, the $\pi$-electron delocalization involving the entire anthracycle is fully equivalent to Clar's $\pi$-sextet migration. Similarly, the higher order $\pi$-circuits in tetracene $(14 \pi$ and $18 \pi)$ and pentacene $(18 \pi$ and $22 \pi)$ also introduce linear dependence to the proposed model and as such they are excluded from considerations. This is especially so in the light of findings by Bultinck et al., ${ }^{7}$ who demonstrated that it is possible to nearly quantitatively reproduce the ring-current maps of a large number of aromatic polycycles by involving $6 \pi-, 10 \pi-$, and $14 \pi$-circuits only. ${ }^{7}$

From an algebraic point of view, the model of migrating $\pi$-circuits relies on a system of linear equations that in the matrix notation, for pentacene, can be compactly represented as follows:

$$
\left(\begin{array}{ccc}
1 & \frac{1}{2} & \frac{1}{3} \\
1 & 1 & \frac{2}{3} \\
1 & 1 & 1
\end{array}\right)\left(\begin{array}{l}
W_{6 \pi} \\
W_{10 \pi} \\
W_{14 \pi}
\end{array}\right)=\left(\begin{array}{c}
R_{\text {Terminal }} \\
R_{\text {Inner }} \\
100 \%
\end{array}\right) .
$$

Here the columns of the $3 \times 3$ matrix collect the linear coefficients related to each of three different patterns of relative aromaticity of rings in pentacene (see the bottom of Figure 1); the $R$-values represent the relative ring aromaticities with respect to the central ring (assumed to be $100 \%$ ) calculated using selected aromaticity criterion, while $W_{6 \pi}, W_{10 \pi}$ and $W_{14 \pi}$ stand for percentage weights of the corresponding $\pi$-electron circuits. Accordingly to the above mentioned arguments, for anthracene and tetracene we assume $R_{\text {Inner }}=$ $100 \%$ and $W_{14 \pi}=0$. Solving Eq. (1) for given $R$-values allows one to determine if and to what extent the inter-ring resonance effects contribute to the global aromaticity of acenes within the framework of particular aromaticity criterion. That is, the greater $W_{6 \pi}$ the lower magnitude of the inter-ring resonance effects and hence the more reliable is the original $\pi$-sextet model by Clar. To illustrate how this simple extension of Clar's concept works in practice we selected six commonly used quantities representing electronic $\left(I_{R}\right.$, PDI, $H_{\mathrm{RCP}}$, and EDDB), structural (HOMA), and magnetic (NICS) criteria of aromaticity; a brief description of these quantities with full references can be found in the review by Feixas et al. ${ }^{8}$ and in our recent comparative studies. ${ }^{9}$ To determine $R$-values we used data from independent benchmark study for all of the above-mentioned descriptors at the density functional theory level $;{ }^{9 a}$ specifically, we chose the $\omega \mathrm{B} 97 \mathrm{X} / \mathrm{cc}-\mathrm{pVTZ}$ method as it provides the most reliable description of electron delocalization and the best (of all tested methods) reproduction of experimental geometries and ionization potentials of all three acenes. ${ }^{9 a}$

The results indicate that, despite apparent qualitative similarities (Figure 2) in local aromaticity trends shown by most of the indices, the calculated contributions of the corresponding $\pi$-electron circuits differ dramatically(Table 1) and divide aromaticity descriptors into two groups. The first group of quantities, i.e. IR, PDI, and especially $H_{\mathrm{RCP}}$, preserves to a large extent the local (benzenelike) nature of aromatic stabilization and seems to only echo the conjugation effects between adjacent rings in all cases (up to about $20 \%$ for pentacene). These indices give the largest weight to $6 \pi$ circuits. This result is in line with the Glidewell-Lloyd rule, ${ }^{5 b}$ an extension of Clar's rule, stating that the total population of $\pi$-electrons in fused conjugated polycyclic systems that have a closedshell singlet ground state tends to form the smallest $4 n+2$ groups. ${ }^{2 b}$ Moreover, the result of the first group of indices may be regarded reasonable as aromaticity indicators like $I_{\mathrm{R}}$ and PDI principally refer only to cyclic delocalization of electrons within particular ring solely (the intra-ring resonance), ${ }^{8}$ and they only indirectly take into account the inter-ring resonance effects, since equilibrium geometries (for which the indices are computed) are determined by the balance between electrostatics, Pauli repulsion, charge transfer, $\sigma$ system contributions, and other effects that may principally have a non-local nature.$^{10}$ All these factors introduce structural differentiation into the consecutive ring positions in acenes making the $\mathrm{C}-\mathrm{C}$ bond lengths more equalized in inner rings rather than in the outer ones. ${ }^{9 a}$ This is particularly confirmed by HOMA, which directly quantifies aromaticity in terms of bond-length alternation. ${ }^{11}$ As shown in Figure 2 and Table 1, HOMA indeed predicts significant diversity of ring aromaticity in acenes (e.g. terminal rings in pentacene are only half as aromatic as the central one). Thus, accordingly to the presented model, the inter-ring resonance effects seem to rule the roost in this case (up to $85 \%$ for pentacene). In fact, HOMA has already been shown by graph-theoretical methods to exhibit a nonlocal characteristic in polycyclic aromatic systems. ${ }^{11}$ But the most surprising fact is that, despite methodological differences, also NICS $(1)_{z z}$ and EDDB show nearly the same magnitude of non-local resonance contributions. On average, aromaticity indices from the second row in Figure 2 predict predominating role of the inter-ring resonance effects from about $60 \%$ in anthracene through $75 \%$ in tetracene up to $85 \%$ in the case of pentacene. Admittedly, indices like NICS $(1)_{z z}$ tend to be criticized severely as aromaticity measures owing to their proven non-local character caused by mixing of different ring currents. ${ }^{13,14}$ But, since HOMA and EDDB (by 

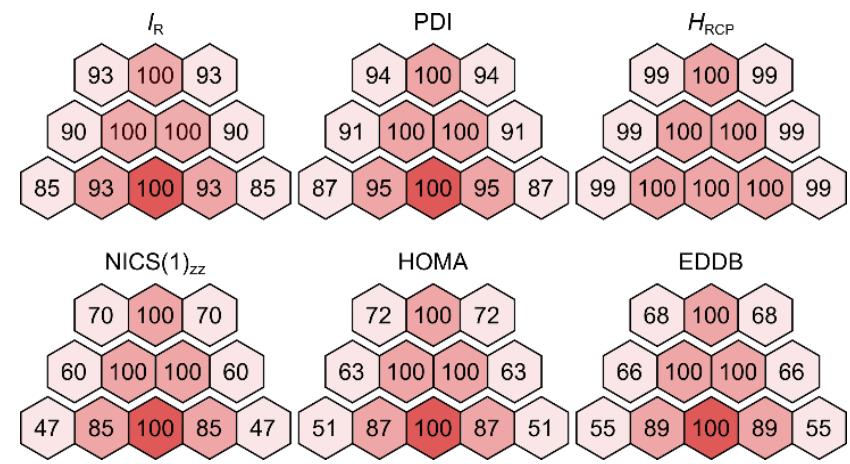

Figure 2. Relative ring aromaticities $(R)$ based on different aromaticity criteria. Computational data taken from Ref. 9a.

Table 1. The percentages of individual $\pi$-circuits obtained by solving Eq. (1) with $R$-values taken from Figure 2.

\begin{tabular}{cccccccc} 
& \multicolumn{2}{c}{ Anthracene } & \multicolumn{2}{c}{ Tetracene } & \multicolumn{3}{c}{ Pentacene } \\
Index & $W_{6 \pi}$ & $W_{10 \pi}$ & $W_{6 \pi}$ & $W_{10 \pi}$ & $W_{6 \pi}$ & $W_{10 \pi}$ & $W_{14 \pi}$ \\
\hline$I_{\mathrm{R}}$ & 86 & 14 & 80 & 20 & 77 & 2 & 21 \\
PDI & 88 & 12 & 82 & 18 & 79 & 6 & 15 \\
$H_{\mathrm{RCP}}$ & 98 & 2 & 98 & 2 & 98 & 2 & 0 \\
NICS $(1)_{z z}$ & 40 & 60 & 20 & 80 & 9 & 46 & 45 \\
HOMA & 44 & 56 & 26 & 74 & 15 & 46 & 39 \\
EDDB & 36 & 64 & 32 & 68 & 21 & 46 & 33
\end{tabular}

definition, a global measure of aromaticity and resonance $)^{9}$ quantitatively confirm predominant role of the inter-ring effects in acenes, the non-locality manifestations by NICS $(1)_{z z}$ cannot be further regarded only as a methodological artifact, ${ }^{14}$ at least in the case of acenes.

The experiment seems to be in favor of the second group of aromaticity indicators. For instance, let us focus on the pentacene molecule. Apart from structural and energetic properties discussed elsewhere, ${ }^{9 a}$ the experimental ${ }^{1} \mathrm{H}$ NMR spectrum obtained by $\mathrm{Na}-$ gano et $a l .{ }^{15}$ reveals that the aromatic protons in pentacene are shifted downfield for about $3.7 \mathrm{ppm}$ (central ring), $3.3 \mathrm{ppm}$ (inner rings) and $1.9 \mathrm{ppm}$ (terminal rings) ${ }^{15 \mathrm{~b}}$ from the reference chemical shift of non-aromatic protons in 1,3-cyclobutadiene (on average, $\left.\delta_{\mathrm{H}, a v}=5.5 \mathrm{ppm}\right) .{ }^{15 \mathrm{c}}$ Solving Eq. (1) with these particular numbers (appropriately normalized to the corresponding $R$-values) gives $W_{6 \pi}=13 \%, W_{10 \pi}=54 \%$, and $W_{14 \pi}=33 \%$, which perfectly agrees with the theoretical predictions by NICS $(1)_{z z}$, HOMA, and EDDB. But even more impressive evidence for the overwhelming non-local character of the resonance effects in pentacene is provided by image of pentacene from the constant-height atomic force microscopy (AFM) obtained by Gross et al. ${ }^{16}$ Admittedly, it has been demonstrated many times that the AFM as well as the scanning tunneling microscopy (STM) images of polycyclic aromatic hydrocarbons show patterns that resemble very much the unique Clar aromatic structures (i.e., rings with localized Clar $\pi$-sextets are indeed better marked in the STM/AFM images). ${ }^{17}$ But as shown in Figure 3 , within the AFM image of pentacene the most visible are the outer edges of terminal rings suggesting strong localization of electrons in these fragments (c.f. the AFM image of hexabenzenocoronene). ${ }^{17 \mathrm{f}}$ It turns out that this characteristic pattern can be rationalized within the framework of the recently proposed electron density partitioning scheme, ${ }^{18 a}$ in which one extracts from the total molecular density $\operatorname{ED}(r)$ the components representing localized bonds, $\operatorname{EDLB}(r)$, and delocalized ones, $\operatorname{EDDB}(r)$. The latter function is congeneric with the EDDB index and provides a global view on the resonance effects in molecular systems. ${ }^{18}$ Even a cursory look at the $\operatorname{EDLB}(r)$ and $\operatorname{EDDB}(r)$ maps (calculated at the same theory level as the aromaticity descriptors) presented in Figure 3, shows that all the inter-ring contributions to resonance concern mainly the central anthracyclic unit, while the outermost rings preserve to a large extent the electronic structure of two well localized double bonds. A striking resemblance between the experimental AFM image and the calculated $\operatorname{EDLB}(r)$ map, supported by the results collected in Table 1, leaves no doubt that inside the pentacene molecule electrons are delocalized over much larger distances than a single hexagon. Furthermore, along with expansion of the volume occupied by the electrons delocalized through the inter-ring resonance between central ring and the inner ones, one should also expect an increase of polarizability in this area. ${ }^{19}$ Indeed, theoretical calculations based on the DFT concept of the so called local softness $S(r),{ }^{20}$ depicted in Figure 3, clearly show that in pentacene carbon atoms in the central anthracycle (and especially in the central ring) are the most reactive centers in the entire molecule, at least in the context of the maximum hardness principle..$^{20}$ In the light of the experimental data, this is directly confirmed by the fact that the central ring in pentacene (but also in anthracene and tetracene) is protonated, adds bromine, and undergoes Diels-Alder reactions readily. ${ }^{5}$ Other authors have attributed the larger reactivity of the central ring of pentacene to the generation of an extra $\pi$-sextets (and also an extra $\pi$-dectet in our model) in the final adduct. ${ }^{2 \mathrm{a}, 5 \mathrm{~b}, \mathrm{~d}, \mathrm{f}}$

To summarize, in this work we have demonstrated using the model of migrating $\pi$-circuits that the local aromaticity concept, intuitively connected with cyclic delocalization of electrons, is actually of minor importance in the context of structural properties and reactivity of $[n]$ acenes $(n=3,4,5)$. The results of theoretical calculations supported by experimental data provide irrefutable evidences for Clar's $\pi$-sextets migration in these systems, and clearly show that the link between the theoretical concept of aromaticity and the real electronic structure (revealed by the constant-height AFM imaging technique) entails separation of intra- and inter-ring resonance effects, which in the case of acenes comes down to solving a system of simple linear equations.

\section{ASSOCIATED CONTENT}

The computational data used in this work were taken entirely from Supporting Information associated with the Reference 9a.

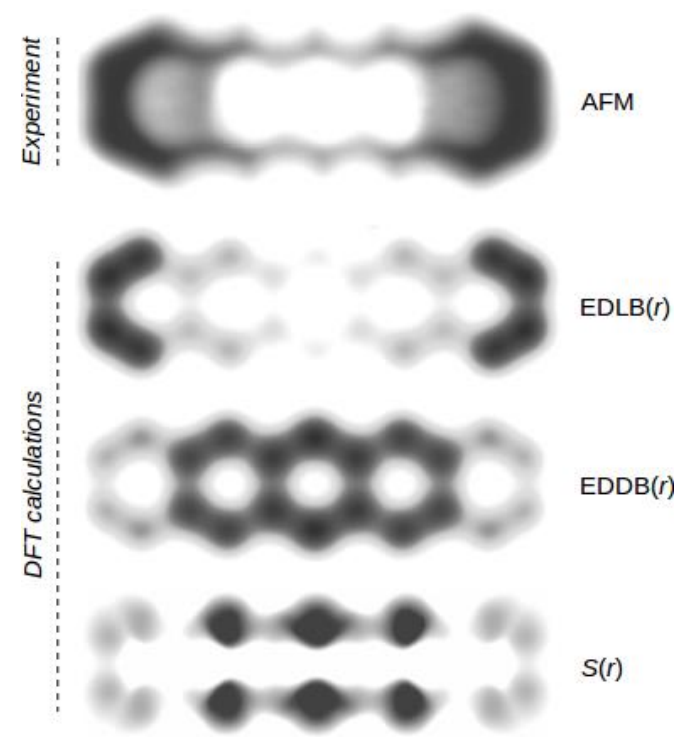

Figure 3. "Images" of pentacene obtained from experiment and quantum-chemical calculations involving $\pi$-electron density. The AFM image reproduced from Ref. 16 with permission from AAAS. 


\section{AUTHOR INFORMATION}

Corresponding Author

*Email: dariusz.szczepanik@uj.edu.pl or dszczpnk@gmail.com.

ORCID

D.W. Szczepanik: 0000-0002-2013-0617

M. Solà: 0000-0002-1917-7450

H. Szatylowicz: 0000-0002-7034-6985

J. Dominikowska: 0000-0003-4150-0506

Author Contributions

All authors have given approval to the final version of the manuscript.

\section{ACKNOWLEDGMENT}

The research was supported in part by the National Science Centre, Poland (NCN SONATA, grant 2015/17/D/ST4/ 00558, DS) as well as the PL-Grid Infrastructure of the Academic Computer Centre CYFRONET with the calculations performed on the cluster platform "Prometheus". Calculations were also partially carried out using resources provided by the Wroclaw Centre for Networking and Supercomputing (http://wcss.pl), grant No. 118 (JD). MS thanks for the support of the Ministerio de Economì y Competitividad of Spain (Project CTQ2017-85341-P), Generalitat de Catalunya (project number 2014SGR931, Xarxa de Referència en Quìmica Teòrica i Computacional, and ICREA Academia prize), and European Fund for Regional Development (FEDER grant UNGI10-4E801). HS thanks the Warsaw University of Technology for supporting this work. JD acknowledges financial support from the National Science Centre of Poland (Grant No. 2015/19/B/ST4/01773).

\section{REFERENCES}

(1) (a) Clar, E. Polycyclic Hydrocarbons; Academic Press: London, 1964. (b) Clar, E. The Aromatic Sextet; Wiley: New York, NY, 1972. (2) (a) Solà, M. Front. Chem. 2013, 1, 22. (b) El Bakouri, O.; Poater, J.; Feixas, F.; Solà; M. Theor. Chem. Acc. 2016, 135, 205.

(3) (a) Schleyer, P. v. R.; Manoharan, M.; Jiao, H.; Stahl, F. Org. Lett. 2001, 3, 3643. (b) Bultinck, P.; Ponec, R.; Carbo-Dorca, R. J. Comput. Chem. 2007, 28, 152. (c) Bultinck, P. Faraday Discuss. 2007, 135, 347. (d) Fowler, P. W.; Myrvold, W. J. Phys. Chem. A 2011, 115, 13191. (e) Portella, G.; Poater, J.; Bofill, J. M.; Alemany, P.; Solà, M. J. Org. Chem. 2005, 70, 2509; Erratum, íbid. 2005, 70, 4560.

(4) (a) Jug, K.; Koster, A. M. J. Am. Chem. Soc. 1990, 112, 6772. (b) Anusooya, Y.; Chakrabarti, A.; Pati, S. K.; Ramasesha, S. Int. J. Quantum Chem. 1998, 70, 503. (c) Cyrański, M. K.; Stepien, B. T.; Krygowski, T. M. Tetrahedron 2000, 56, 9663. (d) Poater, J.; Fradera, X.; Duran, M.; Solà, M. Chem. Eur. J. 2003, 9, 400. (e) Poater, J.; Duran, M.; Solà, M.; Silvi, B. Chem. Rev. 2005, 105, 3911. (f) Bultinck, P.; Ponec, R.; Damme, S. V. J. Phys. Org. Chem. 2005, 18, 706. (g) Bultinck, P.; Ponec, R.; Gallegos, A.; Fias, S.; Damme, S. V.; CarbóDorca, R. Croat. Chem. Acta 2006, 79, 363. (h) Bultinck, P.; Rafat, M.; Ponec, R.; Gheluwe, B. V.; Carbó-Dorca, R.; Popelier, P. J. Phys. Chem. A 2006, 110, 7642.

(5) (a) Altschuler, L; Berliner, E. J. Am. Chem. Soc. 1966, 88, 5837. (b) Glidewell, C.; Lloyd, D. Tetrahedron 1984, 40, 4455. (c) Wiberg, K. B. J. Org. Chem. 1997, 62, 5720. (d) Dabestani, R.; Ivanov, I. N. Photochem. Photobiol. 1999, 70, 10. (e) Steinter, E.; Fowler, P. W. Chem. Phys. Lett. 2002, 364, 259. (f) Chien, S. H.; Cheng, M. F.; Lau, K. C.; Li, W. K. J. Phys. Chem. A 2005, 109, 7509. (g) Zade, S. S.; Bendikov, M. J. Phys. Org. Chem. 2012, 25, 452.

(6) Randić, M. Chem. Rev. 2003, 103, 3449.
(7) (a) Fias, S.; Fowler, P.; Delgado, J. L.; Hahn, U.; Bultinck, P. Chem. Eur. J. 2008, 14, 3093. (b) Fias, S.; Damme, S. V.; Bultinck, P. J. Comput. Chem. 2008, 29, 358. (c) Fias, S.; Damme, S. V.; Bultinck, P. J. Comput. Chem. 2010, 31, 2286.

(8) Feixas, F.; Matito, E.; Poater, J.; Solà, M. Chem. Soc. Rev. 2015, $44,6434$.

(9) (a) Szczepanik, D. W.; Solà, M.; Andrzejak, M.; Pawełek, B.; Dominikowska, J.; Kukułka, M.; Dyduch, K.; Krygowski, T. M.; Szatylowicz H. J. Comput. Chem. 2017, 38, 1640. (b) Szczepanik, D. W.; Andrzejak, M.; Dominikowska, J.; Pawełek, B.; Krygowski, T. M.; Szatylowicz, H.; Solà, M. Phys. Chem. Chem. Phys. 2017, 19, 28970.

(10) Thirman, J.; Engelage, E.; Huber, S. M.; Head-Gordon, M. Phys. Chem. Chem. Phys. 2018, 20, 905.

(11) Krygowski, T. M.; Szatylowicz, H.; Stasyuk, O. A.; Dominikowska, J.; Palusiak, M. Chem. Rev. 2014, 114, 6383.

(12) (a) Ciesielski, A. Krygowski, T. M.; Cyrański, M. K. J. Chem. Inf. Model. 2008, 48, 1358. (b) Ciesielski, A.; Krygowski, T. M.; Cyrański, M. K.; Dobrowolski, M. A.; Aihara, J-I. Phys. Chem. Chem. Phys. 2009, 11, 11447. (c) Ciesielski, A.; Krygowski, T. M.; Cyrański, M. K.; Balaban, A. T. Phys. Chem. Chem. Phys. 2011, 13, 3737. (d) Makino, M.; Nishina, N.; Aihara, J-I. J. Phys. Org. Chem. 2017, doi: $10.1002 /$ poc. 3783 .

(13) (a) Schleyer, P. v. R.; Maerker, C.; Dransfeld, A.; Jiao, H.; Eikema-Hommes, N. J. R. J. Am. Chem. Soc. 1996, 118, 6317. (b) Chen, Z.; Wannere, C. S.; Corminboeuf, C.; Puchta, R.; Schleyer, P. v R. Chem. Rev. 2005, 105, 3842.

(14) (a) Lazzeretti, P.; Prog. Nucl. Magn. Reson. Spectrosc. 2000, 36, 1. (b) Gomez, J. N. A. F.; Mallion, R. B. Chem. Rev. 2001, 101, 1349. (c) Lazzeretti, P.; Phys. Chem. Chem. Phys. 2004, 6, 217. (d) Badri, Z.; Pathak, S.; Fliegl, H.; Rashidi-Ranjbar, P.; Bast, R.; Marek, R. Foroutan-Nejadand, C.; Ruud, K. J. Chem. Theory Comput. 2013, 9 , 4789. (e) Poater, J.; Bofill, J. M.; Alemany, P.; Solà, M. J. Org. Chem. 2006, 71,1700

(15) (a) Nagano, M.; Hasegawa, T.; Myoujin, N.; Yamaguchi, J.; Itaka, K.; Fukumoto, H.; Yamamoto, T.; Koinuma, H. Jpn. J. Appl. Phys. 2004, 43, L315. (b) from two signals, i.e. at 7.4 and $8.0 \mathrm{ppm}$, we selected the former as the latter showed a significant correlation with signals from other rings within the Nuclear Overhauser Effect (NOE) $\begin{array}{llll}\text { measurement. } & \text { (c) } & \text { Chemical Book. }\end{array}$ https://www.chemicalbook.com/SpectrumEN_106-99-0_1HNMR.htm (accessed Dec 25, 2017).

(16) Gross, L.; Mohn, F.; Moll, N.; Liljeroth, P.; Meyer, G. Science 2009, 325, 1110.

(17) (a) Iyer, V. S.; Yoshimura, K.; Enkelmann, V.; Epsch, R.; Rabe, J. P.; Müllen, K. Angew. Chem. Int. Ed. 1998, 37, 2696. (b) Ito, S.; Herwig, P. T.; Böhme, T.; Rabe, J. P.; Rettig, W.; Müllen, K. J. Am. Chem. Soc. 2000, 122, 7698. (c) Samorí, P.; Fechtenkötter, A.; Jäckel, F.; Böhme, T.; Müllen, K.; Rabe, J. P. J. Am. Chem. Soc. 2001, 123, 11462. (d) Samorí, P.; Severin, N.; Simpson, C. D.; Müllen, K.; Rabe, J. P. J. Am. Chem. Soc. 2002, 124, 9454. (e) Watson, M. D.; Jäckel, F.; Severin, N.; Rabe, J. P.; Müllen, K. J. Am. Chem. Soc. 2004, 126, 1402. (f) Gross, L.; Mohn, F.; Moll, N.; Schuler, B.; Criado, A.; Guitián, E.; Peña, D.; Gourdon, A.; Meyer, G. Science 2012, 337, 1326. (g) Gutman, I.; Tomovic, Z.; Mullen, K.; Rabe, J. P. Chem. Phys. Lett. 2004, $397,412$.

(18) (a) Szczepanik, D. W. Comput. Theor. Chem. 2016, 1080, 33. (b) Szczepanik, D. W.; Zak, E. J.; Dyduch, K.; Mrozek, J. Chem. Phys. Lett. 2014, 593, 154. (c) Szczepanik, D. W.; Andrzejak, M.; Dyduch, K.; Zak, E. J.; Makowski, M.; Mazur, G.; Mrozek, J. Phys. Chem. Chem. Phys. 2014, 16, 20514. (d) Szczepanik, D. W. Comput. Theor. Chem. 2017, 1100, 13.

(19) Anslyn, E.; Dougherty, D. Modern Physical Organic Chemistry. University Science, 2006.

(20) (a) Torrent-Sucarrat, M.; De Proft, F.; Ayers, P. W.; Geerlings, P. Phys. Chem. Chem. Phys. 2010, 12, 1072. (b) Parr, R. G.; Chattaraj, P. K. J. Am. Chem. Soc. 1991, 113, 1854. 


\section{FOR TABLE OF CONTENTS ONLY}

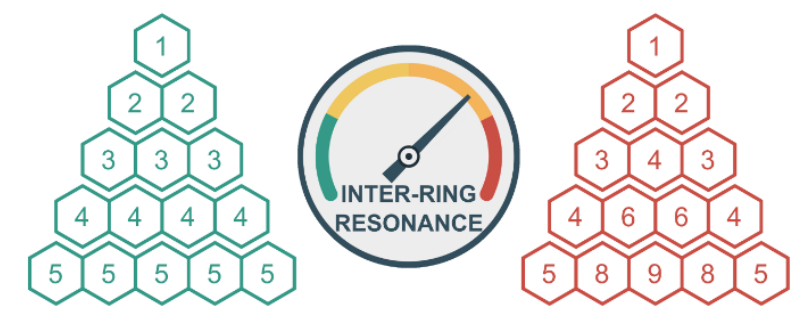

Model of migrating $\pi$-sextets

Model of migrating $\pi$-circuits 